\title{
Themes of the upcoming LEPH2018 Conference
}

\author{
Nick Crofts, AM*
}

\section{OVERARCHING THEME}

\section{Disparities in Health and Criminal Justice}

Health states are intimately related to socioeconomic status, which itself relates to the major direct determinants of health (including access to and quality of health care, social and physical environments, and health behaviours) and further indirect determinants (such as inequality in access to and quality of education, income inequality, and occupational environment). Access to justice and outcomes of involvement with the criminal justice system are also intimately related to socio-economic status and class. Both disparities affect the same communities in ways that are inextricably linked. Therefore, both disparities must be addressed jointly.

Health differences adversely affecting socially disadvantaged groups are particularly unacceptable because ill health can be an obstacle to overcoming social disadvantage ... It is time to be explicit that the heart of a commitment to addressing health disparities is a commitment to achieving a more just society (Bravemean, Kumanyika, Fielding et al, 2011).

Global shifts are taking place in the culture and orientation of law enforcement agencies that have seen their role as purely focused on public safety, specifically crime combat and maintenance of public order, but are increasingly beginning to understand the inextricable links between public safety and public health. In part this has resulted from deliberations about 'community policing', 'problem-solving policing', 'joined-up policing' and 'smart policing'. Being responsive and forward-thinking has led police agencies, particularly in the Global North, to consider the range of social and economic issues that underpin insecurity and risk. Police are now engaging as nodal actors in the governance of both public safety and public health; how this is configured varies according to existing resources, skills, and network actors.

\section{SUBSTANTIVE THEMES}

\section{Crises \& Catastrophes}

This theme covers a very wide range of types of events in which health authorities and law enforcement must respond in a concerted manner and often in the worst of circumstances: natural disasters, major disease outbreaks and pandemics, mass violence, human-caused disasters, technological disasters and, very topically, refugee crises. Adverse health outcomes following these events include injury and death, mental health and physical health problems, drug and alcohol use, and increased mental health service demand. In addition, community safety is threatened by looting, destruction of property, and theft. Evacuations and food and water relief also call for cooperation and collaboration between health and law enforcement.

\section{Violence}

Violence causes major public health outcomes including massive health care service utilization with emergency room attendance and hospitalization, ensuing disability, and death. It is impossible to estimate the lifetime health impact of violence, as exposure to violence as a child can increase health risks in later life. Violence is contagious, inter-generationally reproduced, and shows one of the strongest inequalities gradients. By adopting a public health approach, violence can be prevented, and a wide range of interventions are available to public health practitioners. Violence prevention is a critical element in tackling other public health issues. There is a strong evidence base behind public health approaches to violence prevention with collaborative multi-sectoral approaches led by police and public health authorities.

The LEPH conferences have, to date, focused on domestic and family violence and gender-based violence. With the first appearance of the LEPH conference in the Americas, gun violence becomes a major focus for LEPH2018-12 of the top 15 countries ranked in terms of per capita gun deaths are in the Americas.

\section{Mental Health}

There is a disproportionate involvement of people with serious mental illnesses and in mental health crises in the criminal justice system: 10-30 per cent of all police contacts involve people with mental illnesses, and high proportions of prisoners in all jurisdictions have a mental illness. Mental health-related calls can and do result in police or persons with mental illness being seriously or fatally wounded. At the same time, mental health budgets are being cut in many countries, and many low- and middle-income countries have no, or only rudimentary, community-based mental health services. As mental health services decline, the police role as gatekeepers to both the mental health and criminal justice systems becomes increasingly important; as a result, police see themselves as being relied on as an emergency mental health service. Police perceive mental health-related calls as 
very unpredictable and dangerous which, without adequate training in de-escalation, can rapidly escalate to disastrous ends. Police often do not feel adequately trained to effectively respond to mental health crises, see mental health calls as very time-consuming that divert officers from other crime fighting activities. There is also a feeling that mental health providers are sometimes not sufficiently responsive.

The collaboration of police and mental health service providers has become critical to appropriately serving the needs of individuals experiencing mental health crises and their families and communities.

\section{4. 'Hidden in Plain Sight'}

Health and criminal justice systems frequently encounter people experiencing communication difficulties. This may occur in a wide range of situations, such as when someone goes missing, or are victims, witnesses or offenders of crimes. The needs of the individual may be unclear or misunderstood; the individual may be inappropriately placed and susceptible to repeat harm. Understanding the health/police intersect in support of people experiencing communication problems has become critical in the provision of timely and appropriate risk assessment and safeguarding interventions.

This theme covers a very wide range of events where health services and law enforcement agencies support people, their families, and communities at times when communication is challenging. This may include contact with people who have dementia, learning difficulties, epilepsy, autism, neurobiological brain injury such as stroke or head trauma, hearing or sight impairment, or when someone is unresponsive through injury. Research and innovation in this area of health care is emerging, particularly within the field of speech and language therapy, yet there are opportunities to share and develop multi-agency learning and research in this crucial area of practice. In this theme, we encourage presentations from a range of disciplines to support understandings of communication innovations, joint practices, and research to reduce barriers to effective communications, and improve health and police responses to those in need of communication support. We are particularly interested in exploring how such learning could be transferable and made available within police and health practice and multi-agency safeguarding to maximize opportunities for appropriate communication interventions.

\section{Indigenous Communities}

Providing health services and policing services to Indigenous communities present unique challenges. Indigenous peoples in Canada, US, Australia, and New Zealand face very similar justice and health issues, with lower life expectancies, overrepresentation in the courts and corrections and significant health issues, including higher rates of alcohol and other drug use, mental health issues, and infectious diseases including HIV. Levels of violence are disproportionately higher among Indigenous people, especially intimate partner violence, and alcohol-related violence is a major health concern. Common contributing factors include denial of land rights, remoteness of communities, cultural differences, unemployment and discrimination, poor access to health and other services, poverty, and homelessness. These issues are generally well-researched and well-known, yet they persist.
While whole-of-government responses are required to significantly improve these conditions, the police and public health services often struggle to provide services relevant to Indigenous communities. The conference looks to engage with Indigenous leadership to better understand Indigenous concepts of justice, which includes broader social justice, and collaborative approaches to these highly complex issues, with public health and law enforcement working together with communities.

\section{Vulnerable Populations}

Vulnerability, just as with human fragility and suffering, is universal. Most police interactions are with people who are vulnerable and, as a consequence, vulnerability is increasingly addressed as a rule rather than as an exception in public health and law enforcement procedures. Positioning vulnerability at the centre of policing and public health aligns with many theoretical and applied considerations of disadvantage and how to manage it. A recent focus of commentators, practitioners, and scholars on matters of vulnerability in law enforcement and public health has been in the recognition that vulnerability comes in different shapes and forms, and most importantly, that vulnerabilities can be standalone or multiple, layered, temporary or persistent, or even transgenerational. This theme of the conference looks at the various ways to operationalize vulnerability in law enforcement and public health processes, and the various means to ward off or disrupt the more enduring, persistent, incremental or transgenerational forms of vulnerability.

\section{Alcohol \& Other Drugs}

Both licit and illicit drugs can be associated with adverse health outcomes and with crime and risks to public safety. Police have been critical actors in the reduction of alcoholrelated road trauma and, in collaboration with health authorities, can ameliorate much of the damage caused by alcohol. With illicit drugs too, the police role in supporting the proven public health approach of harm reduction to achieve common goals is critically important and requires a good understanding of the dynamics of drug markets, and of drug use and dependence, as well as a mutually respectful partnership between police and harm reduction programs. An example of a beneficial police role is in the administration of naloxone to people suffering an opioid overdose.

Policies regarding currently illicit drugs vary worldwide and are evolving in different countries and regions-sometimes rapidly. Decriminalization and legalization of cannabis has occurred in many places, some which previously had immensely restrictive and punitive policies on cannabis. The police role clearly changes with changing policy-but in what ways? And where are the guidelines for police in the context of these changes? These are all topics the conference will examine, seeking better understanding of optimum partnerships between law enforcement and the harm reduction, drug treatment, and primary care sectors.

\section{Wellness \& Resilience}

Police are expected to cope with a myriad of complex and often deeply troubling circumstances, reinforced by informal police cultures which promote a type of machismo that does not allow for expressions of vulnerability. However, 
very little attention is given to police officer well-being and resilience. Police are often viewed as resilient when they may simply have developed ways of coping that ultimately lead to burnout and post-traumatic stress disorder. It is not surprising, therefore, that the levels of use of alcohol and other drugs among police officers are high, according to the limited research on this subject. A number of other factors contribute to police vulnerability and compromised wellness and, as part of the society in which they are located, police are perhaps more vulnerable than other professionals to communicable diseases including sexually transmitted infections-particularly true of police in the Global South.

If law enforcement agencies are to operate optimally, deliberations and research focused on the challenges to police officer wellness and resilience are critical. This requires police agencies taking bold steps to empathically uncover the extent of compromised well-being amongst its members, and to ensure that help-seeking is encouraged and enabled. This is important for the organizational health of law enforcement agencies. But equally important, the police are more likely to be empathic to those who are most vulnerable to public health threats if police members are willing to acknowledge that they are affected by these issues too.

In this theme, we encourage presentations on the difficulties that law enforcement agencies, and the individuals that constitute them, face in regard to wellness and resilience. In so doing, a space will be provided for participants at the conference to deliberate on what services and processes could be made available to the police to maximize wellness and resilience. The conference also provides a forum for exploring the legitimate fears that law enforcement officers have in policing vulnerable population groupings.

\section{Corrections-Prisons as Public Health Institutions}

In most societies at most times, people who are incarcerated are disproportionately from lower social classes and have disproportionately increased health-especially mental health-needs. The provision of appropriate and high-quality health care to prisoners is critically important for pragmatic reasons, as part of rehabilitation, but more so to meet their human rights and address social inequities which compound their marginalization. But, as well, we must look at the role prisons play in the health of the wider community and address the role of prisons and imprisonment in spreading disease and impairing health and resilience. The HIV epidemic has been a classic example, where the role of prisons in the epidemic has been regularly described as being 'incubators' and 'mixing machines'. Prisons in many societies act as de facto mental health institutions, but usually with few or no mental health services-a situation which aggravates the impact of mental illness on the whole community.

Ernie Drucker in A Plague of Prisons (2013) makes the case that our current unprecedented level of imprisonment has become an epidemic, and argues that imprisonment-originally conceived as a response to the crimes of individuals-has become mass incarceration: a destabilizing force, a plague upon our body politic, that undermines families and communities, damaging the very social structures that prevent crime. We seek in this theme to examine the public health role of prisons and ways to improve their impact on the public health of their society.
We must examine the public health impact of intensifying incarceration legislation - both within and beyond prison walls. .... although confirming associations between marginalization and disease is important, we equally and urgently need to improve the health and health care of those most at risk, with the hope of reducing the burden of HIV and HCV in the community.

An emerging field ...

\section{Epidemiological Criminology (Conceptual Development \& Methodology)}

Those working in the fields of criminal justice and public health share a concern with understanding and reducing risky behaviours and environments. Yet ironically, it is only recently that these have engaged in strategic, interdisciplinary conversations to integrate theory and methods toward innovative policy, programmatic, and scientific solutions. This convergence can be depicted in the 'Epidemiological Criminology' paradigm, which encompasses efforts to understand and evaluate the nexus between crime and health in the context of behavioural change and analysis. Such efforts include the scientific study of risk and protective factors that transcend disciplinary, geospatial, and ecological boundaries.

Epidemiological criminology subsumes the most innovative strands in criminological and public health theories and methods, including experimental criminology and environmental criminology-in dialogue with acute care and preventative medicine, public health practice and behavioural change, public health law research, and social epidemiology. From an epidemiological perspective, a central policy question for law enforcement practitioners and researchers is whether (and how) police officers (and the criminal justice system, broadly) can influence behaviours and environments in ways that address threats to public health while, at the same time, disrupting and preventing criminal behaviour. The 'downstream' effects of policing policy and practice on both the functioning and wider health impacts and health disparities of the criminal justice system are also subsumed under this emerging paradigm.

For this theme, presentations on innovative theoretical or methodological developments at the intersection of epidemiology and criminology are encouraged. Examples include, but are not limited to, data on the geographic patterning of crime and health-related incidents, or the presentation of new analytic techniques and theories for interpreting the spatial (and/or social) relationships between health risk behaviours and criminal behaviour. Theoretical papers might address the influence of public health laws and/or criminal laws on policing policy and practice, or the ways in which community-based, problem-oriented policing (e.g., "Smart Policing") interventions can or could impact the social determinants of health and crime. Other topics that bridge theories and methods in public health, policing, criminology, and criminal justice systems are encouraged.

\section{Economics of Policing (empirical research as related to policy-making LEPH)}

The Economics of Policing strives to understand the social and financial costs and benefits of policing by looking at the efficiency, effectiveness, and equity of police service delivery and policing models. In examining these factors, 
it is important to understand the context and complexity of policing-that is, the external factors impacting policing, relationships with the public, with other public and private service providers, legislation and the courts, and with governments-and within that understanding, to develop methods of costing, measuring performance, understanding the multi-disciplinary aspects of policing, and understanding sustainability. Policing does not operate independently of the public or of other government services; therefore, collaborative approaches are needed to effectively contribute to public safety and community well-being.

\section{Policing and Public Health Education and Training Agenda}

Despite frequent aspirational calls for cross-sectoral collaboration, public health and safety professionals and institutions continue to work in silos, and-at times-at cross-purposes. Numerous factors impede integration, including divergent professional cultures, skill-sets, and incentives, as well as the pervasive demand to do more with less. Education and training serves as an additional barrier, but is also one of the key building blocks towards improved collaboration. Achieving police/public health synergy requires an agenda to reform training, skill-building, and other educational infrastructure within these sectors, at both frontline and management levels. This includes developing a shared vocabulary, service systems, and key performance indicators, which can then be integrated into law enforcement and public health training curriculums. If police officers continue to take on an increasing role as public health interventionists and collaborators, then we also must start building a theoretical and practicumbased framework for better education. What pedagogical, technological, and other tools can be deployed to deliver new training content in most effective and cost-effective ways?

Academic public health education also needs to include the importance and role of law enforcement, especially police, in the public health mission. This theme emphasizes the importance of such training and education in bringing law enforcement and public health practitioners together in their pursuit of healthy and safe communities.

\section{CONFLICT OF INTEREST DISCLOSURES}

The author declares there are no conflicts of interest.

\section{AUTHOR AFFILIATIONS}

*University of Melbourne, Melbourne, AZ.

\section{REFERENCES}

Braveman, P.A., Kumanyika, S., Fielding, J., LaVeist, T., Borrell, L.N. Manderscheid, R., Troutman, A., et al. (2011). Health disparities and health equity: The issue is justice. Am J Public Health, 101(Suppl 1), S149-S155.

Drucker, E. (2013). A plague of prisons: The epidemiology of mass incarceration in America. New York, NY: The New Press.

\section{SUGGESTED READINGS}

Akers, T.A., Potter, R.H., \& Hill, C.V. (2013). Epidemiological criminology: A public health approach to crime and violence. San Francisco, CA: John Wiley and Sons.

Bartkowiak-Théron, I.M.F., \& Asquith, N.L. (2017). Conceptual divides and prac tice synergies in law enforcement and public health: Some lessons from policing vulnerability in Australia. Policing and Society, 27(3), 276-288.

Bellis, M., Hughes, K., Perkins, C., \& Bennett, A. (2012). Protecting people, promoting health: A public health approach to violence prevention for England. Liverpool, UK: North West Public Health Observatory.

Cowan-Dewar JC, Kendall C, \& Palepu A. (2011). Prisons and public health Open Medicine, 5(3), el32-133.

Julian, R., Bartkowiak-Théron, I., Hallam, J., \& Hughes, C. (2017). Exploring law enforcement and public health as a collective impact initiative: Lessons learned from Tasmania as a case study. Journal of Criminological Research, Policy and Practice, 3(2)

Waltermaver, E., \& Akers, T.A., (Eds). (2013). Epidemiological criminology: Theory to practice. New York, NY: Routledge.

Watson, A.C., Fulambarker, A.J. (2012). The crisis intervention team model of police response to mental health crises: A primer for mental health practitioners. Best Pract Ment Health, 8(2), 71. 